\title{
El láser como coadyuvante en el tratamiento de las úlceras
}

\author{
Eneritz Diez Alcorta( ${ }^{(1)}$, Naiara Ibarra Diez ${ }^{(2)}$ \\ (1) Fisioterapeuta, Hospital de Galdakao-Usansolo. \\ (2) Enfermera, UAP OSI-Barrualde.
}

\section{Correspondencia:}

Naiara Ibarra Diez.

aizerrota@hotmail.com

\section{RESUMEN}

Entre el $75 \%$ y el $80 \%$ de las úlceras de la extremidad inferior son de etiología venosa. Además, constituyen un gasto económico sanitario elevado, lo que nos lleva a plantear la necesidad de encontrarles una solución costo-efectiva.

Las úlceras venosas tratadas con láser de baja potencia evolucionan satisfactoriamente en un corto espacio de tiempo, lo que demuestra la efectividad de dicho tratamiento aunque todavía no hay suficiente evidencia que avale su uso.

El principal objetivo de este estudio era mostrar la eficacia del láser en el tratamiento de úlceras de difícil cicatrización. Para ello, se presenta el caso de una mujer que presentaba úlceras venosas de 5 años de evolución en ambas extremidades inferiores que no habían mejorado con los cuidados de enfermería habituales.

Tras uno y seis meses respectivamente de tratamiento conjunto enfermería-fisioterapia, ambas lesiones cicatrizaron.

\section{PALABRAS CLAVE}

Skin ulcer; Low-level light therapy; Nursing.

\section{INTRODUCCIÓN}

Una úlcera cutánea es una pérdida de sustancia que afecta a la epidermis, la dermis y, en ocasiones, a planos más profundos, con extensión, forma y profundidad variable (1).

Una herida crónica es una lesión de la piel con una escasa o nula tendencia a la cicatrización mientras se mantenga la causa que la produce (2). Se considera que una herida se cronifica cuando no ha culminado el proceso de cierre de la misma en un período de 6 semanas (3)

Las úlceras pueden ser(2) por presión, por humedad, arteriales, neuropáticas, neoplásicas, venosas o por otras causas.

Las úlceras venosas son debidas a un inadecuado retorno venoso que provoca una insuficiencia venosa que desemboca en una hipertensión venosa y finalmente en úlcera.

Según los datos extraídos de la Conferencia Nacional de Consenso sobre Úlceras de la Extremidad Inferior (CONUEI) se estima que la prevalencia de úlceras de extremidades inferiores es de entre $0,10 \%$ y $0,30 \%$ con una incidencia de 3 a 5 casos nuevos por mil personas y año (4). Estos datos deben multiplicarse por dos cuando se considere el segmento poblacional de más de 65 años (4). Entre el $75 \%$ y el $80 \%$ de las úlceras de la extremidad inferior son de etiología venosa siendo su prevalencia entre el 0,5\% y 0,8\% y su incidencia de 2 a 5 casos nuevos por mil personas y año (4).

La aplicación de compresión externa inicia diversos efectos fisiológicos y bioquímicos complejos que afectan a los sistemas venoso, arterial y linfático. Siempre que el nivel de compresión

no afecte adversamente al flujo arterial y se apliquen las técnicas y materiales correctos, los efectos de la compresión pueden ser drásticos, reduciendo el edema y el dolor a la vez que favorecen la curación de úlceras causadas por insuficiencia venosa (5).

Las úlceras venosas de extremidades inferiores constituyen un gasto económico sanitario elevado (4-6), lo que nos lleva a plantear la necesidad de encontrar una solución costo-efectiva.

Las úlceras venosas tratadas con láser de baja potencia evolucionan satisfactoriamente en un corto espacio de tiempo, lo que demuestra la efectividad de dicho tratamiento(7-8-9-10). La palabra láser es el acrónimo de "Light amplification by stimulated emission of radiation"(8).

Algunas de las características de la luz láser son las siguientes (7-8-9):

- No divergencia: La luz normal se dispersa 360ำ, pero en el proceso de obtención del láser se depura el haz de tal forma que solo salga en una determinada dirección.

- Monocromatismo: Todos los fotones de un mismo aparato son iguales, y eso hace que cada haz sea de una sola longitud de onda y, por tanto, se observe un solo color.

El cuerpo humano tiene una serie de receptores de ondas como los ojos (luz visible) los oídos (sonidos) y los receptores táctiles de vibración. Las células de nuestro organismo están preparadas para obtener energía del medio, incluída la radiación infrarroja de la luz solar. 
En fisioterapia se utiliza un tipo de láser de baja intensidad, en inglés Low Intensity Laser Therapy (LILT) o Low Level Laser Therapy (LLLT) (11), el cual, no calienta la zona a tratar de forma detectable (7).

El tratamiento de heridas abiertas y úlceras representa la aplicación fundamental de los aparatos de láser de baja intensidad. (7) La fotobioactivación(la estimulación de diversos eventos biológicos que utilizan energía de la luz pero sin cambios significativos de temperatura $\left.{ }^{(11)}\right)$ es un término de uso común en relación con LILT. La investigación ha demostrado que el láser frio puede estimular estructuras intracelulares y sus funciones" (7-8-9-11-1213-14-15-16-17-18).

El láser aporta energía para que las células optimicen el proceso de cicatrización. Aunque aún queda mucho por estudiar sobre qué longitud de onda es la ideal para favorecer la cicatrización de heridas, en fisioterapia se utilizan longitudes de onda entre 600 y 1064 nm, dependiendo del método de obtención del láser y de la forma de aplicación (7). Las dosis bibliografiadas consensuadas para el tratamiento de heridas son entre 4 y 10 julios/ $\mathrm{cm} 2$ (7), pero en lesiones generales de tejidos blandos se admiten dosis de 30-40 J/cm2 (7) siempre y cuando la frecuencia de aplicación no sea mayor a una vez por día de tratamiento. Además el láser también es efectivo en el tratamiento del dolor (7-8-14-16-17) que a veces acompaña a este tipo de heridas.

El presente trabajo se planteó, como principal objetivo, mostrar la eficacia del láser en el tratamiento de una paciente que presentaba dos úlceras venosas de difícil cicatrización.

\section{MATERIAL Y MÉTODO}

Se presenta el siguiente caso, que fue tratado en lo que actualmente es la Organización de Servicios Integrados de Osakidetza (OSI-Barrualde), concretamente en una de las consultas de enfermería de atención primaria de esta OSI, en colaboración con la unidad de fisioterapia del Hospital de Galdakao-Usansolo.

Caso clínico: Mujer de 65 años de edad, con hipertensión arterial, obesidad e insuficiencia venosa que presentaba úlceras venosas de 5 años de evolución en ambas extremidades inferiores que no habían mejorado con los cuidados de enfermería habituales. Había sido valorada por cirugía vascular en el 2008, sin seguimiento ni tratamiento por su parte. Pasó a estar a cargo de la enfermera que realiza este trabajo en mayo del 2010. En ese momento se le realizó una valoración global del estado de salud y se inició educación sanitaria para que perdiera peso. Asímismo, se valoró el sistema vascular: mantenía pulsos periféricos y no presentaba signos de infección.

Al inicio del tratamiento la paciente presentaba las lesiones que se detallan a continuación:

Extremidad Inferior Izquierda:

- Úlcera de aproximadamente $9 \mathrm{~cm} \times 6 \mathrm{~cm}$ con signos de contaminación crítica, bordes mal delimitados y piel perilesional dañada

\section{Extremidad Inferior Derecha:}

- Úlcera grande (10 cm x 5 cm en sus zonas más larga y ancha), en la zona anterior-media de la pierna

- úlcera pequeña $(1 \mathrm{~cm} \times 1 \mathrm{~cm})$, encima del maléolo interno. Reabierta y resistente al tratamiento

En ese momento se realizaban curas con hidrogel para posibilitar el desbridamiento, y como apósito secundario espuma de poliuretano sin reborde, que gestionaba bien el exudado y aseguraba una retirada poco traumática. Además se realizaba vendaje de compresión en ambas extremidades.

En el mes de Julio se observaron signos de infección (coloración más amarillenta del lecho de las heridas, aumento del dolor, mal olor, etc). Se comenzó tratamiento antibiótico oral según antibiograma y a nivel local se empezó a usar apósito de hidrofibra de hidrocoloide con plata. Una vez controlado el brote infeccioso se reajustó el tipo de cura y se realizaron cultivos de control para descartar nuevos brotes.

Como el tamaño de las heridas no disminuía y la paciente refería mucho dolor se decidió añadir terapia láser al tratamiento.

El 1 de septiembre del 2010 comienza la colaboración enfermería-fisioterapia. Para aplicar el tratamiento láser la fisioterapeuta necesita levantar el apósito de la cura, con lo cual los cuidados de enfermería deben ser también diarios y posteriores al tratamiento fisioterápico. En todo momento se mantuvo la compresión terapéutica.

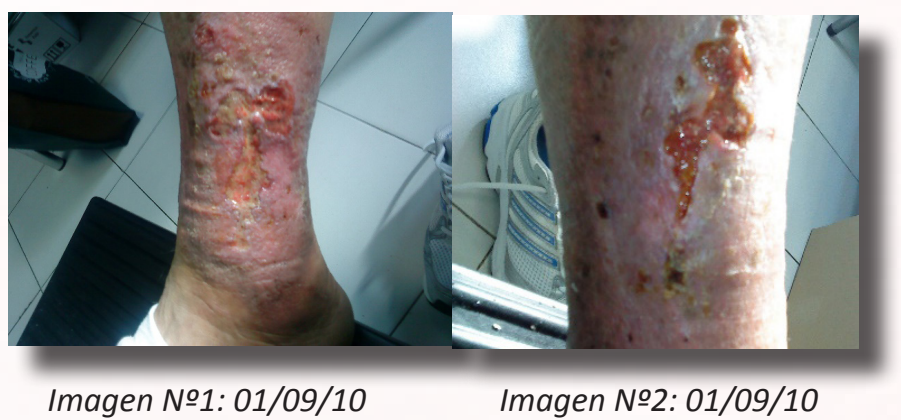

Como la úlcera es una herida abierta y no debe manipularse más de lo estrictamente necesario, el método de elección de aplicación de la luz láser es un cañón, que crea un rayo de luz que mediante un juego de espejos puede dirigirse a la zona a tratar.

Consideraciones especiales en el uso del láser: Paciente y profesionales deben cubrirse los ojos con gafas especiales para evitar quemaduras. No debe aplicarse en embarazadas (7-9) ni en pacientes con cáncer(8-9). En colecciones infecciosas o en heridas infectadas tampoco debe aplicarse si el proceso se manifiesta como florido, pero en procesos larvados frecuentemente los tejidos reciben el empuje necesario para la reactivación del sistema defensivo(8).

En este caso se decidió aplicar láser infrarrojo de baja intensidad, con una longitud de onda de $795 \mathrm{~nm}, 5$ veces por semana, con una dosis de 25 julios $/ \mathrm{cm}^{2}$ de herida (con un láser de ca- 
ñón se delimitó la zona que iba a recibir el tratamiento, que fue aplicado en forma de barrido de manera que cada centímetro cuadrado de herida recibiese la misma cantidad de energía). La decisión del uso de estos parámetros se tomó teniendo en cuenta la antigüedad de la lesión y el intenso dolor que presentaba la paciente en ese momento.

\section{RESULTADOS}

Tras 79 sesiones y 6 meses de tratamiento conjunto la herida de la extremidad inferior izquierda cicatrizó por completo (Imágenes 3-4).

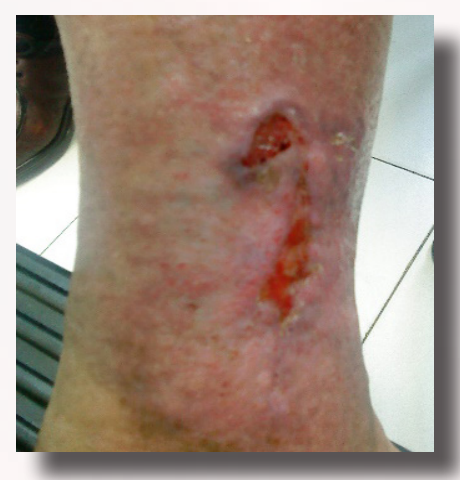

Imagen №3: 22/10/10

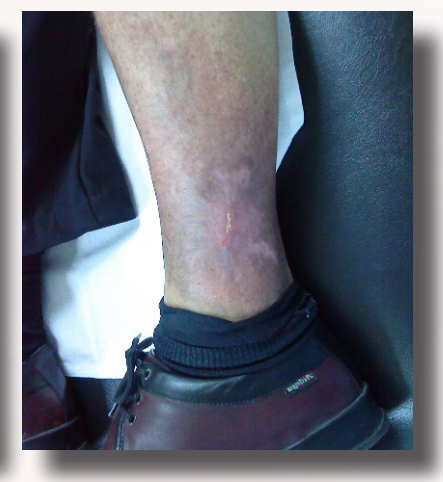

Imagen №4: 03/02/11
Tras realizar 27 sesiones de tratamiento conjunto fisioterapia-enfermería las heridas de la extremidad inferior derecha cicatrizaron (Imágenes 5-6).

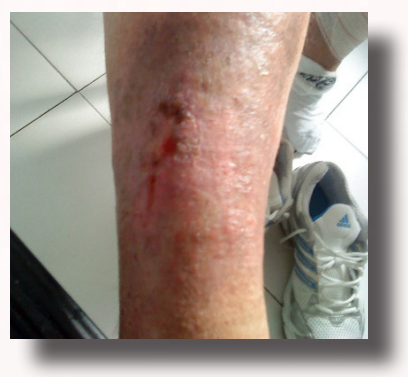

Imagen No5: 22/09/10

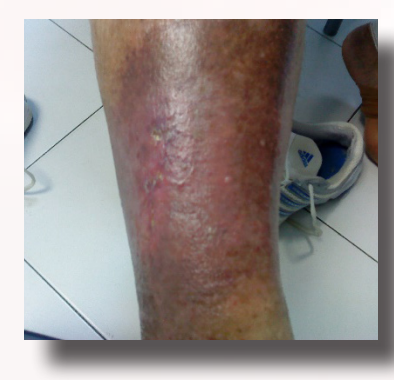

Imagen №6: 15/10/10
Después de casi 7 años desde el comienzo del tratamiento las úlceras permanecen cerradas. En la Ell se desarrolló una nueva úlcera en abril de 2014, tras herida traumática, pero se resolvió satisfactoriamente tras realizar nuevo tratamiento conjunto.

El tratamiento, desde entonces y hasta la fecha de redacción del presente artículo (Junio de 2016), ha sido resolutivo y no se han requerido sesiones adicionales de tratamiento fisioterápico ni otros cuidados de enfermería; sólo se han pautado autocuidados como vigilancia diaria de la integridad de la piel, secado correcto de las extremidades inferiores, hidratación de las mismas y uso de medias de compresión de forma continua durante el día para evitar recidivas.

\section{DISCUSIÓN Y CONCLUSIONES}

En este caso, la actuación conjunta de enfermería, con sus cuidados, y fisioterapia, con el uso del láser, ha hecho posible la cicatrización de unas úlceras que no respondían al tratamiento habitual.
El láser de baja intensidad parece una herramienta eficaz en el tratamiento de úlceras de difícil cicatrización, pero hacen falta más estudios de magnitud en este campo que aporten mejor evidencia científica, ya que hasta el momento solo hay referencias a experiencia clínica.

\section{AGRADECIMIENTOS}

Queremos mostrar nuestro más sincero agradecimiento a todas las personas que, gracias a su apoyo, han permitido que este trabajo fuera posible:

A doña Arantza Zabala, jefa del servicio de fisioterapia del Hospital de Galdakao, por facilitar el tratamiento de nuestra paciente en las instalaciones de las cuales ella es responsable; al equipo médico rehabilitador de dicho hospital, especialmente al doctor don Francisco Javier López de Muniain, médico que se hizo cargo de la paciente de este caso clínico; a doña Maria Teresa Intxausti, auxiliar y colaboradora por su inestimable ayuda y a doña Belén Diez Alkorta, microbióloga y profesora, por su lectura crítica y aportaciones en la elaboración del trabajo escrito.

Y a todo el equipo de fisioterapeutas y auxiliares, por facilitar nuestro trabajo diario, por ser fuente de conocimiento y apoyo, pero sobre todo, por enseñarnos que el bienestar del paciente es lo primero. Gracias.

\section{BIBLIOGRAFÍA}

1. Servicio Madrileño de Salud. Recomendaciones para el tratamiento local de las úlceras cutáneas crónicas de la Comunidad de Madrid. Madrid: Comunidad de Madrid. Consejería de Sanidad; 2010.

2. García-Fernández, FP, López-Casanova, P, Segovia-Gómez, T,Soldevilla-Agreda JJ, Verdú Soriano, J. Unidades multidisciplinares de Heridas Crónicas: Clinicas de heridas. Serie de documentos de posicionamiento GNEAUPP no 10. Grupo Nacional Para el Estudio y Asesoramiento en Úlceras por Presión y Heridas Crónicas. Logroño 2012.

3. Cacicedo González R, Castañeda Robles C, Cossio Gómez F, Delgado Uría A, et all. Manual de Prevención y Cuidados Locales de Heridas Crónicas. Servicio Cántabro de Salud. 1o Edición. Enero 2011.

4. Conferencia Nacional de Consenso sobre ulceras de la extremidad inferior (CONUEI). Documento de Consenso de CONUEI. Madrid. Edikamed. [internet]2009. Disponible en: http://www. aeev.net/guias/CONUEI2009.pdf

5. Chow RT, Johnson MI, Lopes-Martins RA, Bjordal JM. Efficacy of low-level laser therapy in the management of neck pain: a systematic review and meta-analysis of randomised placebo or active-treatment controlled trials. Lancet. 2009; 374: 18971908.doi: 10.1016/S0140-6736(09)61522-1.

6. Purwins S, Herberger K, Debus ES, Rustenbach SJ, Pelzer $P$, Rabe $E$, et al. Cost-of-illness of chronic leg ulcers in Germany. Int Wound J. 2010; 7 (2): 97-102. doi: 10.1111/j.1742481X.2010.00660.x. 
7. Baxter GD. Tratamiento con láser de baja intensidad. Electroterapia: Practica basada en la evidencia. 12a ed. España: Elsevier.2009; 161-177.

8. Rodríguez Martín JM. Electroterapia en fisioterapia. Ed Panamericana. 2014: 569-631.

9. Khan J. Principios y Practica de Electroterapia. Ed Jims.1991; 57-77.

10. De la Cruz Fornaguera Y, Del Olmos Pimentel D, Quiñones Castro M, Zulueta Salazar A. Comportamiento de las úlceras venosas de los miembros inferiores tratadas con láser de baja potencia. Rev Cubana de Angiologia y Cirugia Vascular. 2012;13 (1).

11. Watson T. Laser Therapy. Electrotherapy on the web [Internet]. UK; c1995-2017 [Consultado 17-03-2017]. Disponible en: http://www.electrotherapy.org/modality/laser-therapy

12. Laakso L, Richardson C, Cramond T. Factors affecting Low Level Laser Theraphy. Australian Journal of Physiotherapy. 1993; 39 (2):95-99.

13. Gaida K, Koller R, Isler C, Aytekin O, Al-Awami M, Meisi G, Frey M. Low level laser therapy: a conservative approach to the burns scar?. Burns. 2004; 30 (4): 362-367.

14. Yamany A.A, Sayed H. M. Effect of low laser level therapy on neurovascular function of diabetic peripheral neurophaty. Journal of Advanced Research. 2012; 3:21-28.

15. Bie RA, DE Vet HCW, Lenssen TF, Van den Wildenberg FAJM, Koostra G, Knipschild PG. Low Level Laser Therapy in ankle sprains: a randomized clinical trial. Arch Phys Med Rehabil 1998;79:1415-1420.
16. Djavid GE, Mehrdad R, Ghasemi M, Hasan-Zadeh H, Sotoodeh-Manesh A, Pouryaghoub G. In chronic low back pain, Low Level Laser Therapy combined with exercise is more benefitial tan exercise alone in the long term: a randomised trial. Australian Journal of Physiotherapy 2007; 52: 155-160.

17. Bjordal JM, Couppé C, Chow RT, Tunér J, Ljunggren AE. A systematic review of Low Level Laser Therapy with location-specific doses for pain from chronic joint disorders. Australian Journal of Physiotherapy. 2003; 49: 107-116.

18. Allegra C, Nelson A, Rabe E, Soldevilla JJ, Torra JE, Vin F. Comprendiendo la terapia compresiva. Documento de posicionamiento EWMA. [internet]. 2003. Disponible en: http://studylib. es/doc/5273054/comprendiendo-la-terapia-compresiva

19. Guía de práctica clínica para el cuidado de personas que sufren quemaduras. Servicio Andaluz de Salud. Consejería de Salud. Junta de Andalucía. [internet] 2011. Disponible en: http://www. guiasalud.es/GPC/GPC 485 Quemados Junta Andalucia completa.pdf

20. Salvador Sanz J.F, Novo Torres, A, Lorda Barraguer, E, Castillo, F, Torra i Bou, J.E, Torregrosa Ramos, M.J. Estudio comparativo de efectividad de un apósito de plata nanocristalina frente a sulfadiazina argéntica en el tratamiento de pacientes quemados. Cirugía Plástica Ibero-Latinoamericana. 2011; 37(3): 253-266. 